\title{
Mapping bijectively $\sigma$-algebras onto power sets
}

\author{
N. Kwami Agbeko
}




\title{
MAPPING BIJECTIVELY $\sigma$-ALGEBRAS ONTO POWER
}

\section{SETS}

\author{
N. Kwami Agbeko \\ Department of Applied Mathematics, University of Miskolc \\ 3515 Miskolc - Egyetemváros, Hungary \\ matagbek@gold.uni-miskolc.hu \\ [Received February 6, 2001] \\ Dedicated to the memory of my Father
}

\begin{abstract}
As an application of the so-called "optimal measure" we attempt to seek sets whose power sets are equinumerous with $\sigma$-algebras, which seems to be new information about $\sigma$-algebras.
\end{abstract}

Mathematical Subject Classification: 28A20, 28E10

Keywords: Measurable sets

\section{Introduction}

Some new information about $\sigma$-algebras is investigated, consisting of mapping bijectively $\sigma$-algebras onto power sets. Such $\sigma$-algebras, in fact, form a rather broad class. A special grouping of the so-called optimal measures is used in our investigation (for more about optimal measures cf. [1-4]. We provide constructively a bijective mapping that will serve the purpose. In the proof we first characterize set-inclusion as well as some asymptotic behaviors of sequences of measurable sets. Without loss of generality we shall restrict ourselves to infinite $\sigma$-algebras, since the opposite case can be easily done.

Throughout this communication $(\Omega, \mathcal{F})$ will stand for an arbitrary measurable space, with both $\Omega$ and $\mathcal{F}$ being infinite sets (where, as usual, the elements of $\mathcal{F}$ are referred to as measurable sets).

By an optimal measure we mean a set function $p^{*}: \mathcal{F} \rightarrow[0,1]$ which fulfills the following axioms:

P1. $p^{*}(\emptyset)=0$ and $p^{*}(\Omega)=1$.

P2. $p^{*}(B \bigcup E)=p^{*}(B) \bigvee p^{*}(E)$ for all measurable sets $B$ and $E$ (where $\bigvee$ stands for the maximum).

P3. $p^{*}\left(\bigcap_{n=1}^{\infty} E_{n}\right)=\lim _{n \rightarrow \infty} p^{*}\left(E_{n}\right)=\bigwedge_{n=1}^{\infty} p^{*}\left(E_{n}\right)$, for every decreasing sequence of measurable sets $\left(E_{n}\right)$, where $\bigwedge$ stands for the minimum.

In [2] we have obtained the following results for all optimal measures $p^{*}$. 
By $\left(p^{*}\right)$-atom we mean a measurable set $H, p^{*}(H)>0$ such that whenever $B \in \mathcal{F}$, $B \subset H$, then $p^{*}(B)=p^{*}(H)$ or $p^{*}(B)=0$.

A $p^{*}$-atom $H$ is decomposable if there exists a subatom $B \subset H$ such that $p^{*}(B)=$ $p^{*}(H)=p^{*}(H \backslash B)$. If no such subatom exists, we shall say that $H$ is indecomposable.

Fundamental Optimal Measure Theorem. Let $(\Omega, \mathcal{F})$ be a measurable space and $p^{*}$ an optimal measure on it. Then there exists a collection $\mathcal{H}\left(p^{*}\right)=\left\{H_{n}: n \in J\right\}$ of disjoint indecomposable $p^{*}$-atoms, where $J$ is some countable (i.e. finite or countably infinite) index-set such that for any measurable set $B$, with $p^{*}(B)>0$, we have that

$$
p^{*}(B)=\max \left\{p^{*}\left(B \bigcap H_{n}\right): n \in J\right\} .
$$

Moreover, the only limit point of the set $\left\{p^{*}\left(H_{n}\right): n \in J\right\}$ is 0 provided that $J$ is a countably infinite set. $\left(\mathcal{H}\left(p^{*}\right)\right.$ is referred to as a $p^{*}$-generating countable system.)

\section{NOTATIONS.}

1. $\mathcal{P}$ will denote the set of all optimal measures defined on $(\Omega, \mathcal{F})$.

2. $\mathcal{P}_{\infty}$ is the set of all optimal measures whose generating systems are countably infinite.

3. For every $A \in \mathcal{F}$, we write $\bar{A}$ for the complement of $A$.

4. $\mathbb{N}$ stands for the set of counting numbers (or positive integers).

5. $A \subset B$ means set $A$ is a proper subset of set $B$.

6. $A \subseteq B$ means set $A$ is a subset of set $B$.

7. $\mathbb{P}(A)$ stands for the power set of set $A$.

\section{Main results}

Definition 2.1. We say that an optimal measure $p^{*} \in \mathcal{P}_{\infty}$ is of order-one if there is a unique indecomposable $p^{*}$-atom $H$ such that $p^{*}(H)=1$. (Any such atom will be referred to as an order-one-atom and the set of all order-one optimal measures will be denoted by $\widetilde{\mathcal{P}_{\infty}^{1}}$.)

Example 1. Fix a sequence $\left(\omega_{n}\right) \subset \Omega$ and define $p_{0}^{*} \in \mathcal{P}_{\infty}$ by

$$
p_{0}^{*}(B)=\max \left\{\frac{1}{n}: \omega_{n} \in B\right\} .
$$

Then $p_{0}^{*} \in \widetilde{\mathcal{P}_{\infty}^{1}}$.

In fact, via the Structure Theorem, there is an indecomposable $p_{0}^{*}$-atom $H$ such that $p_{0}^{*}(H)=1$. This is possible if and only if $\omega_{1} \in H$. We note that there is no other indecomposable $p_{0}^{*}$-atom $H^{*}$ with $H^{*} \cap H=\emptyset$ such that $p_{0}^{*}\left(H^{*}\right)=1$, otherwise necessarily it would ensue that $\omega_{1} \in H^{*}$, which is absurd. Hence $p_{0}^{*} \in \widetilde{\mathcal{P}_{\infty}^{1}}$.

\section{FURTHER NOTATIONS.}

If $H$ is the order-one-atom of some $p^{*} \in \widetilde{\mathcal{P}_{\infty}^{1}}$, we write $p=\left\{q^{*} \in \widetilde{\mathcal{P}_{\infty}^{1}}: q^{*}(H)=1\right\}$. We then refer to the elements of the class $p$ as representing members of the class, and 
call $H$ the unitary atom of the class. (If the unitary atom of a class is the order-oneatom of a representing member, we shall speak of representation.)

We further denote by $\mathcal{P}_{\infty}^{1}$ the set of all $p$ classes.

If $A$ is a nonempty measurable set and $p \in \mathcal{P}_{\infty}^{1}$, the identity $p(A)=1$ (resp. the inequality $p(A)<1$ ) will simply mean that $p^{*}(A)=1$ (resp. $p^{*}(A)<1$ ) for any representing member $p^{*} \in p$. We shall also write $p(A)=0$ to mean that $p^{*}(A)=0$ whenever $p^{*} \in p$.

Write $\nabla$ for the set of all unitary atoms on the measurable space $(\Omega, \mathcal{F})$.

Lemma 2.1. Let $A, B \in \mathcal{F}$ and $p \in \mathcal{P}_{\infty}^{1}$ be arbitrary. In order that $p(A \cap B)=1$ it is necessary and sufficient that $p(A)=1$ and $p(B)=1$.

Proof. As the necessity is obvious, we only have to show the sufficiency. In fact, assume that $p(A)=1$ and $p(B)=1$. Let $H$ be the unitary atom of class $p$, and let $p^{*}$ denote an arbitrary but fixed representing member in the class. Without loss of generality we may assume that $p^{*}$ is a representation of $p$ (i.e. $H$ is the order-one atom of $\left.p^{*}\right)$. Then $p^{*}(H)=1$. Clearly, $p^{*}(A \cap H)=1$ and $p^{*}(B \cap H)=1$. Hence $p^{*}(\bar{A} \cap H \cap \bar{B})=0$. It is enough to prove that both identities $p^{*}(A \cap H \cap \bar{B})=0$ and $p^{*}(\bar{A} \cap H \cap B)=0$ are valid. On the contrary, assume that at least one of these identities fails to hold: $p^{*}(A \cap H \cap \bar{B})=0$, say. Then $p^{*}(A \cap H \cap \bar{B})=1$. Now, since $p^{*}(H \cap B)=1$, it ensues that either $p^{*}(A \cap H \cap B)=1$ or $p^{*}(\bar{A} \cap H \cap B)=1$. Then combining each of these last identities with $p^{*}(A \cap H \cap \bar{B})=1$, we have that $p^{*}(A \cap H \cap \bar{B})=1$ and $p^{*}(A \cap H \cap B)=1$, or $p^{*}(A \cap H \cap \bar{B})=1$ and $p^{*}(\bar{A} \cap H \cap B)=1$. This violates that $H$ is an order-one-atom (because the sets $A \cap H \cap B, A \cap H \cap \bar{B}$ and $\bar{A} \cap H \cap B$ are pairwise disjoint). q.e.d.

Remark 2.0. Let $p \in \mathcal{P}_{\infty}^{1}$ be arbitrary. Then the identity $p(\emptyset)=0$ holds.

Remark 2.1. Let $A \in \mathcal{F}$ and $p \in \mathcal{P}_{\infty}^{1}$ be arbitrary. Then the identities $p(A)=1$ and $p(\bar{A})=1$ cannot hold simultaneously, i.e., for no representing member $p^{*}$ of class $p$ the identities $p^{*}(A)=1$ and $p^{*}(\bar{A})=1$ hold at the same time.

In fact, assume the contrary. Then Lemma 2.1 would imply that

$$
p(A)=p(\bar{A})=1=p(A \cap \bar{A})=p(\emptyset)=0
$$

which is absurd, indeed. q.e.d.

Definition 2.2. For any $A \in \mathcal{F}$ define the set $\Delta(A)$ by

1. $\Delta(A) \subseteq \mathcal{P}_{\infty}^{1}$.

2. If $p \in \Delta(A)$, then $p(A)=1$.

Remark 2.2. Let $A \in \mathcal{F}$. Then $\Delta(A)=\emptyset$ if and only if $A=\emptyset$. 
Remark 2.3. If $H$ is a unitary atom (with $p$ its corresponding class), then $\Delta(H)=$ $\{p\}$.

Let $A \in \mathcal{F}$ and denote by $\nabla_{A}$ the set of all unitary atoms $H$ such that $p(A)=1$, where $\Delta(H)=\{p\}$. It is clear that $\nabla_{A} \bigcap \nabla_{\bar{A}}=\emptyset$ and $\nabla_{A} \bigcup \nabla_{\bar{A}}=\nabla$. From this observation the following lemma is straightforward:

Lemma 2.2. For every set $A \in \mathcal{F}$, we have that $\Delta(\bar{A})=\overline{\Delta(A)}$.

Proposition 2.3. Let $A, B \in \mathcal{F}$ be arbitrary. Then

$$
\begin{aligned}
& \text { 1. } \Delta(\Omega)=\mathcal{P}_{\infty}^{1} . \\
& \text { 2. } \Delta(A \cap B)=\Delta(A) \cap \Delta(B) . \\
& \text { 3. } \Delta(A \cup B)=\Delta(A) \cup \Delta(B) .
\end{aligned}
$$

Proof. Part 1 is an easy task. Let us show Part 2. In fact, let $p \in \Delta(A \cap B)$. Then $p(A \cap B)=1$. Hence Lemma 2.1 implies that $p(A)=1$ and $p(B)=1$, so that $p \in \Delta(A)$ and $p \in \Delta(B)$, i.e. $p \in \Delta(A) \cap \Delta(B)$. Consequently $\Delta(A \cap B) \subseteq$ $\Delta(A) \cap \Delta(B)$. To show the reverse inclusion, pick an arbitrary $p \in \Delta(A) \cap \Delta(B)$. Then $p(A)=1$ and $p(B)=1$. Via Lemma 2.1, we have that $p(A \cap B)=1$, i.e. $p \in \Delta(A \cap B)$. So $\Delta(A) \cap \Delta(B) \subseteq \Delta(A \cap B)$.

To end the proof, let us show the third part. In fact, let $A$ and $B \in \mathcal{F}$ be arbitrary. Then making use of the second part of this proposition, it ensues that $\Delta(\bar{A} \cap \bar{B})=\Delta(\bar{A}) \cap \Delta(\bar{B})$. By applying Lemma 2.2 and De Morgan identities, we obtain that

$$
\begin{aligned}
\Delta(A \cup B) & =\overline{\overline{\Delta(A \cup B)}}=\overline{\Delta(\bar{A} \cap \bar{B})}=\overline{\Delta(\bar{A}) \cap \Delta(\bar{B})} \\
& =\overline{\Delta(\bar{A})} \cup \overline{\Delta(\bar{B})}=\overline{\overline{\Delta(A)}} \cup \overline{\overline{\Delta(B)}}=\Delta(A) \cup \Delta(B) .
\end{aligned}
$$

This was to be proven. q.e.d.

Lemma 2.4. Let $A$ and $B \in \mathcal{F}$ be arbitrary nonempty sets. In order that $A \subset B$, it is necessary and sufficient that $\Delta(A) \subset \Delta(B)$.

Proof. As the necessity is trivial, we need only show the sufficiency. In fact, assume that $A \backslash B$ is not an empty set. Then because of Remark 2.2, $\Delta(A \backslash B)$ is neither empty. Fix some $p \in \Delta(A \backslash B)$, i.e. $p(A \backslash B)=1$. This implies that $p(B)<1$. (Otherwise we would obtain via Lemma 2.1 that $1=p((A \backslash B) \cap B)=p(\emptyset)=0$, which is absurd.) Then $p(A)=1$ and $p(B)<1$, i.e. $p \in \Delta(A) \backslash \Delta(B)$. So the set $\Delta(A) \backslash \Delta(B)$ is not empty. q.e.d.

Lemma 2.5. Let $A$ and $B \in \mathcal{F}$ be arbitrary nonempty sets. In order that $A \cap B=\emptyset$, it is necessary and sufficient that $\Delta(A) \cap \Delta(B)=\emptyset$.

(The proof follows from Proposition 2.3/2 and Remark 2.2.)

Lemma 2.6. Let $A$ and $B \in \mathcal{F}$ be arbitrary nonempty sets. In order that $A=B$ it is necessary and sufficient that $\Delta(A)=\Delta(B)$. 
Proof. As the necessity is trivial, we need only show the sufficiency. In fact, assume that $A$ and $B \in \mathcal{F}$ are such that $\Delta(A)=\Delta(B)$, i.e. $\Delta(A) \subseteq \Delta(B)$ and $\Delta(B) \subseteq$ $\Delta(A)$. By applying twice Lemma 2.4 it ensues that $A \subseteq B$ and $B \subseteq A$. Therefore $A=B$. q.e.d.

Lemma 2.7. Let $A$ and $B \in \mathcal{F}$ be arbitrary nonempty sets. Then $\Delta(A \backslash B)=$ $\Delta(A) \backslash \Delta(B)$.

Proof. We simply note that Proposition 2.3/2 and Lemma 2.2 entail that

$$
\begin{aligned}
\Delta(A \backslash B) & =\Delta(A \cap \bar{B})=\Delta(A) \cap \Delta(\bar{B}) \\
& =\Delta(A) \cap(\overline{\Delta(B)})=\Delta(A) \backslash \Delta(B),
\end{aligned}
$$

which completes the proof. q.e.d.

Proposition 2.8. Let $\left(A_{n}\right) \subset \mathcal{F}$ and $A \in \mathcal{F}$ be arbitrary. Then $\left(A_{n}\right)$ converges increasingly to $A$ if and only if $\left(\Delta\left(A_{n}\right)\right)$ converges increasingly to $\Delta(A)$.

Proof. Assume that $\left(A_{n}\right)$ converges increasingly to $A$. Then by applying repeatedly Lemma 2.4, we have for every $n \in \mathbb{N}$ that

$$
\Delta\left(A_{n}\right) \subset \Delta\left(A_{n+1}\right) \subset \Delta(A) .
$$

We need to prove that $\Delta(A)=\bigcup_{n=1}^{\infty} \Delta\left(A_{n}\right)$. To do this, it will be enough to show that $\Delta(A) \subseteq \bigcup_{n=1}^{\infty} \Delta\left(A_{n}\right)$ and $\bigcup_{n=1}^{\infty} \Delta\left(A_{n}\right) \subseteq \Delta(A)$. In fact, we note that the second inclusion is trivial. To prove the first one, let us pick an arbitrary class $p \in \Delta(A)$ and fix any representing member $p^{*}$ of class $p$. We note that following the proof of Lemma 0.1 (cf. [1], page 134), there can be found a positive integer $n_{0}$ such that $1=p^{*}(A)=p^{*}\left(\bigcup_{k=1}^{\infty} A_{k}\right)=p^{*}\left(A_{n}\right)$, whenever $n \geq n_{0}$. Hence $p \in \bigcup_{n=n_{0}}^{\infty} \Delta\left(A_{n}\right) \subseteq$ $\bigcup_{n=1}^{\infty} \Delta\left(A_{n}\right)$, i.e.

$$
\Delta(A) \subseteq \bigcup_{n=n_{0}}^{\infty} \Delta\left(A_{n}\right) \subseteq \bigcup_{n=1}^{\infty} \Delta\left(A_{n}\right)
$$

Conversely, assume that sequence $\left(\Delta\left(A_{n}\right)\right)$ converges increasingly to $\Delta(A)$. Then for every $n \in \mathbb{N}$ we have that $\Delta\left(A_{n}\right) \subseteq \Delta\left(A_{n+1}\right) \subseteq \Delta(A)$, so that $A_{n} \subseteq A_{n+1} \subseteq A$ (because of Lemma 2.4). Hence $\bigcup_{n=1}^{\infty} A_{n} \subseteq A$. Now, suppose that set $A \backslash \bigcup_{n=1}^{\infty} A_{n}$ is not empty. Then via Remark 2.2 and Axiom 3 there can be found some $p \in \mathcal{P}_{\infty}^{1}$ and some representing member $p^{*}$ of class $p$ such that

$$
1=p^{*}\left(A \backslash \bigcup_{n=1}^{\infty} A_{n}\right)=p^{*}\left(\bigcap_{n=1}^{\infty} A \cap \overline{A_{n}}\right)=\bigwedge_{n=1}^{\infty} p^{*}\left(A \cap \overline{A_{n}}\right),
$$


since sequence $\left(\overline{A_{n}}\right)$ is a decreasing sequence. Consequently $1=p^{*}\left(A \cap \overline{A_{n}}\right)$ for all $n \in \mathbb{N}$. But Lemma 2.1 yields that $p^{*}(A)=1$ and $p^{*}\left(\overline{A_{n}}\right)=1$ for all $n \in \mathbb{N}$. Hence Axiom 3 entails that

$$
1=\bigwedge_{n=1}^{\infty} p^{*}\left(\overline{A_{n}}\right)=p^{*}\left(\bigcap_{n=1}^{\infty} \overline{A_{n}}\right)=p^{*}(\bar{A})
$$

Nevertheless, this contradicts Remark 2.1 q.e.d.

Proposition 2.9. Let $\left(A_{n}\right) \subset \mathcal{F}$ and $A \in \mathcal{F}$ be arbitrary. Then $\left(A_{n}\right)$ converges decreasingly to $A$ if and only if $\left(\Delta\left(A_{n}\right)\right)$ converges decreasingly to $\Delta(A)$.

Proof. Assume that $\left(A_{n}\right)$ converges decreasingly to $A$. Then by applying repeatedly Lemma 2.4, we have for every $n \in \mathbb{N}$ that

$$
\Delta(A) \subset \Delta\left(A_{n+1}\right) \subset \Delta\left(A_{n}\right) .
$$

We need to prove that $\Delta(A)=\bigcap_{n=1}^{\infty} \Delta\left(A_{n}\right)$. To do this, it will be enough to show that $\Delta(A) \subseteq \bigcap_{n=1}^{\infty} \Delta\left(A_{n}\right)$ and $\bigcap_{n=1}^{\infty} \Delta\left(A_{n}\right) \subseteq \Delta(A)$. In fact, we note that the first inclusion is trivial. To prove the second inclusion let us pick some $p \in \bigcap_{n=1}^{\infty} \Delta\left(A_{n}\right)$. Then $p \in \Delta\left(A_{n}\right)$ for all $n \in \mathbb{N}$. Hence $p\left(A_{n}\right)=1$ for all $n \in \mathbb{N}$. If we fix any representing member $p^{*}$ in class $p$, we then obtain via Axiom 3 that

$$
p^{*}(A)=p^{*}\left(\bigcap_{n=1}^{\infty} A_{n}\right)=\bigwedge_{n=1}^{\infty} p^{*}\left(A_{n}\right)=1,
$$

implying that $p(A)=1$, i.e. $p \in \Delta(A)$. Consequently, $\bigcap_{n=1}^{\infty} \Delta\left(A_{n}\right) \subseteq \Delta(A)$.

Conversely, assume that sequence $\left(\Delta\left(A_{n}\right)\right)$ converges decreasingly to $\Delta(A)$. Then for every $n \in \mathbb{N}$ we obtain that $\Delta(A) \subset \Delta\left(A_{n+1}\right) \subset \Delta\left(A_{n}\right)$ so that $A \subset A_{n+1} \subset A_{n}$, $n \in \mathbb{N}$ (by Lemma 2.4). Hence $A \subseteq \bigcap_{n=1}^{\infty} A_{n}$. To show the reverse inclusion let us assume that set $\left(\bigcap_{n=1}^{\infty} A_{n}\right) \backslash A$ is not empty. Then via Remark 2.2 and Axiom 3 there can be found some $p \in \mathcal{P}_{\infty}^{1}$ such that for every representing member $p^{*}$ of class $p$

$$
1=p^{*}\left(\left(\bigcap_{n=1}^{\infty} A_{n}\right) \backslash A\right)=p^{*}\left(\bigcap_{n=1}^{\infty} A_{n} \cap \bar{A}\right)=\bigwedge_{n=1}^{\infty} p^{*}\left(A_{n} \cap \bar{A}\right)
$$

since $\left(A_{n}\right)$ is a decreasing sequence. Consequently, $1=p^{*}\left(A_{n} \cap \bar{A}\right)$ for all $n \in \mathbb{N}$. Hence Lemma 2.1 yields that $p(\bar{A})=1$ and $p\left(A_{n}\right)=1$ for all $n \in \mathbb{N}$. But then $p \in \Delta\left(A_{n}\right)$ for all $n \in \mathbb{N}$ and hence $p \in \bigcap_{n=1}^{\infty} \Delta\left(A_{n}\right)=\Delta(A)$. Nevertheless, this 
is absurd since $p \in \Delta(\bar{A})=\overline{\Delta(A)}$. We can thus conclude on the validity of the proposition. q.e.d.

Theorem 2.10. Let $\left(A_{n}\right) \subset \mathcal{F}$ and $A \in \mathcal{F}$ be arbitrary. In order that $\left(A_{n}\right)$ converge to $A$, it is necessary and sufficient that $\left(\Delta\left(A_{n}\right)\right)$ converge to $\Delta(A)$.

Proof. For every counting number $n \in \mathbb{N}$ write $E_{n}=\bigcap_{k=n}^{\infty} A_{k}$ and $B_{n}=\bigcup_{k=n}^{\infty} A_{k}$. It is clear that sequence $\left(B_{n}\right)$ converges decreasingly to $\lim \sup A_{n}$ and sequence $\left(E_{n}\right)$ converges increasingly to $\liminf _{n \rightarrow \infty} A_{n}$. Consequently, by applying Theorems 2.8 and 2.9 to these sequences, we can conclude on the validity of the theorem. q.e.d.

Definition 2.3. A mapping $\Delta: \mathcal{F} \rightarrow \mathbb{P}\left(\mathcal{P}_{\infty}^{1}\right)$ is said to be powering if it is defined by:

$$
\Delta(A)=\left\{\begin{array}{cc}
\emptyset & \text { if } A=\emptyset \\
\left\{p \in \mathcal{P}_{\infty}^{1}: p(A)=1\right\} & \text { if } A \neq \emptyset
\end{array}\right.
$$

Remark 2.3. If $H$ is the unitary atom of a class $p \in \mathcal{P}_{\infty}^{1}$, then $\Delta(H)=\{p\}$.

The following result can easily be derived from Lemma 2.6 and Remark 2.2.

Proposition 2.11. If $\Delta: \mathcal{F} \rightarrow \mathbb{P}\left(\mathcal{P}_{\infty}^{1}\right)$ is a powering mapping, then it is an injection.

Definition 2.4. If $\Gamma \subseteq \mathcal{P}_{\infty}^{1}$ is a nonempty set, then the collection $\mathcal{C}$ of all the unitary atoms of the classes $p \in \Gamma$ will be called unitary-atomic collection of $\Gamma$.

Postulate of powering. If $\Gamma \in \mathbb{P}\left(\mathcal{P}_{\infty}^{1}\right) \backslash\{\emptyset\}$ and $\mathcal{C}$ denotes the governing-atomic collection of $\Gamma$, then $\bigcup \mathcal{C}$ is measurable and $\Delta(\bigcup \mathcal{C}) \subseteq \Gamma$.

Theorem 2.12. The powering mapping $\Delta: \mathcal{F} \rightarrow \mathbb{P}\left(\mathcal{P}_{\infty}^{1}\right)$ is surjective if and only if the postulate of powering is valid.

Proof. Assume that Postulate of powering is valid. Let $\Gamma \in \mathbb{P}\left(\mathcal{P}_{\infty}^{1}\right)$ be arbitrarily fixed. We note that if $\Gamma=\emptyset$, then there is nothing to be proven. Suppose that $\Gamma$ is a nonempty subset of $\mathcal{P}_{\infty}^{1}$, and denote by $\mathcal{C}$ its corresponding governing-atomic collection. Then $\bigcup \mathcal{C}$ is measurable and $\Delta(\bigcup \mathcal{C}) \subseteq \Gamma$ (by the postulate). Let us show that $\Gamma \subseteq \Delta(\bigcup \mathcal{C})$. In fact, pick any class $p \in \Gamma$ and $p^{*}$ any representing member of $p$, with $H$ the unitary atom of $p$. Since $H \subseteq \cup \mathcal{C}$, it ensues from Lemma 2.2 that $\Delta(H) \subseteq \Delta(\bigcup \mathcal{C})$. But, via Remark 2.3 we have that $\{p\}=\Delta(H)$ and $p \in \Delta(\bigcup \mathcal{C})$, i.e. $\Gamma \subseteq \Delta(\bigcup \mathcal{C})$. Therefore $\Gamma=\Delta(\bigcup \mathcal{C})$.

To prove the converse biconditional, let us assume that the powering mapping $\Delta$ is a surjection. We note that $\Delta$ is a bijection, since it is also an injection (by Proposition 2.11). Let $\Gamma \in \mathbb{P}\left(\mathcal{P}_{\infty}^{1}\right) \backslash\{\emptyset\}$ be arbitrary and write $\mathcal{C}$ for the corresponding unitaryatomic collection. Obviously we have that $\Gamma=\bigcup\{\Delta(H): H \in \mathcal{C}\}$ is a subset of $\mathcal{P}_{\infty}^{1}$. 
Then via the bijective property it ensues that $\Delta^{-1}(\Gamma) \in \mathcal{F}$. Clearly $\Delta(H) \subset \Gamma$ for every $H \in \mathcal{C}$. By Lemma 2.2 together with the bijective property, we obtain that

$$
H=\Delta^{-1}(\Delta(H)) \subset \Delta^{-1}(\Gamma)
$$

whenever $H \in \mathcal{C}$. Consequently the inclusion $\bigcup \mathcal{C} \subseteq \Delta^{-1}(\Gamma)$ follows. Now let us show that if $\omega \in \Delta^{-1}(\Gamma)$, then there is some $H \in \mathcal{C}$ such that $\omega \in H$. Assume on the contrary that there can be found some $\omega_{1} \in \Delta^{-1}(\Gamma)$ such that $\omega_{1} \notin H$ for all $H \in \mathcal{C}$. We can thus define an optimal measure $q^{*}: \mathcal{F} \rightarrow[0,1]$ so that

$$
q^{*}(B) \begin{cases}=1 & \text { if } \omega_{1} \in B \\ <1 & \text { if } \omega_{1} \notin B .\end{cases}
$$

(See Example 1) Then there is a unique indecomposable $q^{*}$-atom (to be denoted by $\widetilde{H})$ such that $q^{*}(\widetilde{H})=1$. It is clear that $\omega_{1} \in \widetilde{H}$ and $q^{*}\left(\Delta^{-1}(\Gamma)\right)=1$. We further note that

$$
\bigcup\{\Delta(H): H \in \mathcal{C}\}=\Gamma=\Delta\left(\Delta^{-1}(\Gamma)\right)=\left\{p \in \mathcal{P}_{\infty}^{1}: p\left(\Delta^{-1}(\Gamma)\right)=1\right\} .
$$

From this fact and the identity $q^{*}\left(\Delta^{-1}(\Gamma)\right)=1$, there must exist some class $p_{0} \in \mathcal{P}_{\infty}^{1}$ with $p_{0}\left(\Delta^{-1}(\Gamma)\right)=1$, such that $q^{*}\left(\widetilde{H} \cap H \cap \Delta^{-1}(\Gamma)\right)=1$, where $H$ is the unitary atom of class $p_{0}$. Nevertheless, this is possible only if $\omega_{1} \in H$, which is absurd, since we have supposed that $\omega_{1} \notin H$ for all $H \in \mathcal{C}$. Therefore, if $\omega \in \Delta^{-1}(\Gamma)$, then there is some $H \in \mathcal{C}$ such that $\omega \in H$. It ensues that $\omega \in \bigcup \mathcal{C}$ for all $\omega \in \Delta^{-1}(\Gamma)$, as $H \subset \bigcup \mathcal{C}$ whenever $H \in \mathcal{C}$. Thus $\Delta^{-1}(\Gamma) \subseteq \bigcup \mathcal{C}$. Therefore, $\bigcup \mathcal{C}=\Delta^{-1}(\Gamma)$, which leads to the postulate. q.e.d.

Theorem 2.12 entails that an infinite $\sigma$-algebra is equinumerous with a power set if and only if Postulate 1 is valid. This suggests that there are infinite $\sigma$-algebras that are not equinumerous with infinite power sets.

\section{REFERENCES}

[1] Agbeko, N. K.: On optimal averages, Acta Math. Hung. 63 (1994) 1-15.

[2] Agbeko, N. K.: On the structure of optimal measures and some of its applications, Publ. Math. Debrecen 46 (1995) 79-87.

[3] Agbeko, N. K.: How to characterize some properties of measurable functions, Mathematical Notes, Miskolc 2 (2000) 87-98.

[4] Fazekas, I.: A note on 'optimal measures', Publ. Math. Debrecen 51/3-4 (1997) 273-277. 